\title{
Overuse injury and growing bones: the young athlete at risk*
}

\author{
David F. Gerrard MB ChB
}

Otago University, School of Physical Education, Dunedin, New Zealand

\begin{abstract}
Increasing numbers of children are becoming involved in competitive sport. International trends in pre-adolescent sports participation are mirrored in New Zealand, where promising young athletes are being exposed to highintensity training from an earlier age. As a consequence, overuse injuries which were traditionally described in more mature athletes are now becoming recognized in pre-adolescents. The immature musculoskeletal system is less able to cope with repetitive biomechanical stress. Sites of overuse injury reflect the sites of rapid musculoskeletal development. It therefore behoves all medical practitioners, but particularly those in primary care, to be aware of the young athlete at risk. Inherent in the presentation of such musculoskeletal insult there often lurks an over-enthusiastic parent. We are all well reminded of the covert pressures adults may bring to bear upon children. Psychological, as well as physical injury often results.
\end{abstract}

Keywords: Children, sport, epiphysis, injury, sports medicine
Over the past two decades in New Zealand, an increasing number of children have joined the competitive sporting ranks ${ }^{1}$. Most authorities agree that such physical pursuits are both beneficial and necessary for normal development, provided that sport can be kept as safe as possible and enjoyable to participants ${ }^{2}$.

The Accident Compensation Corporation participation rates survey (1985), revealed that $503000 \mathrm{New}$ Zealand children aged between 5 and 19 years played organized winter sport. The Life in New Zealand Study, an initiative of the Hillary Commission (1990), stated that there was a dearth of information available on the participation of children in physical activity. The data presented in the study highlighted a gender preference for certain sports (particularly the traditional winter sports of netball, rugby and soccer). Of interest was the high involvement of 11-13-year-old children in non-structured activities such as skate-

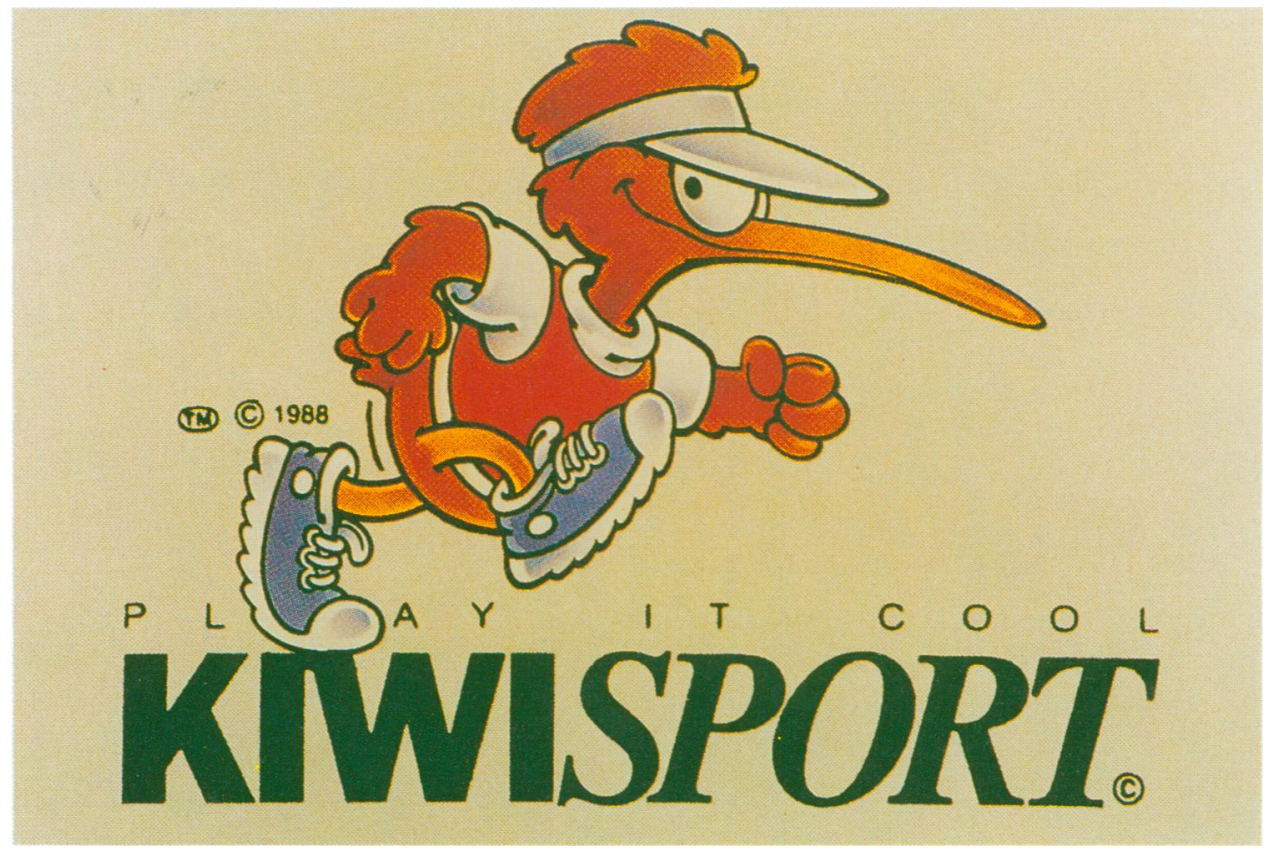

*A paper delivered to the Royal N.Z. College of General Practitioners Conference, Rotorua, July 1992

Address for correspondence: Dr D. F. Gerrard, Otago University, School of Physical Education, Dunedin, New Zealand 
boarding. The popular choices of activity and the numbers participating in physical activity between the ages of 5 and 15 years are illustrated in Figure 1.

In 1988 (again as the result of the influence of the Hillary Commission), this country saw the launch of KiwiSport, a widely applauded initiative which provided the stimulation of modified sport for all children under 12 years of age. In all, 22 sports were catered for under a system of simplified rules, which allowed children of all abilities to participate on equal terms. There was a clear emphasis against competition and a strong emphasis upon enjoyment and skill acquisition.

The success of KiwiSport highlights the importance of introducing pleasure and safety into children's sport. Inherent in this is the implication that many young athletes are having too much too soon. There are compelling psychological as well as physiological reasons for us to view children's sport both broadly and with wisdom.

Given this country's enthusiasm for sport and our international success, the 'catch them young' philosophy ${ }^{3}$ has its adherents in enthusiastic coaches and over-anxious parents. Such vigorous talent identification more often results in psychologically depleted stars (ready to retire in their 'teens), or chronically debilitated victims of overuse injury.

The International Federation of Sports Medicine (FIMS) states ${ }^{4}$ that intensive training of children has no physiological or educational justification, and believes that diversity of movement and all-round physical conditioning should have priority over later specialization. Whereas play and traditional games are involuntary and unstructured, the prime feature of organized sport is the use of systematic repetitive training to improve fitness and develop specialist skills. The New Zealand Federation of Sports Medicine endorses the sentiments of its parent international body by promoting the values of broad non-specialized sports participation for preadolescents.

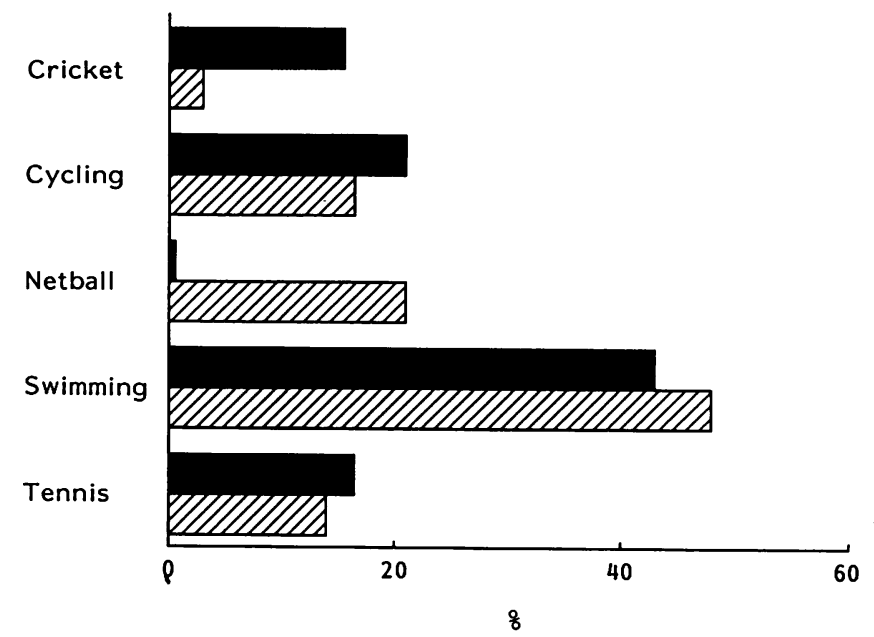

Figure 1. Children's physical activity. Participation rates of New Zealand children between the ages of 5 and 15 years. , boys; girls. Reproduced from Life in New Zealand Survey with permission (C) Hillary Commission 1990

\section{Injury and incidence}

There are no good epidemiological data available in New Zealand to compare the rates of injuries in sport with those of free play activities. In the USA, the boom in organized sport for children and adolescents has been matched by an increase in injuries to areas of active musculoskeletal development $t^{5,6}$.

Injury is the result of two distinct biomechanisms. The first is the result of a single violent impact, the second the end product of many repetitive minor insults. Spontaneous, unstructured activities may well expose children to the major forces of impact and violence in the same way that fractures and dislocations result from organized body contact sport. But the minor, individually insignificant stresses which accumulate to produce overuse syndromes are more obviously linked to established sporting patterns. It has become clear, that the young athlete is at risk from microtrauma which may be sports specific.

Reports of 6-year-old marathoners, 8-year-old swimmers churning out $20 \mathrm{~km}$ per day in the pool, young tennis players hitting hundreds of serves and volleys, and young gymnasts spending hours perfecting their routines are recorded in the sports medical literature?

The FIMS statement ${ }^{4}$ confirms the establishment of a new genre of paediatric sports injury. Overuse injuries such as these were totally unknown in children until the advent of repetitive sports training.

Today there is a growing list of common sites of childhood sports injury, each known either by its eponym or the sport with which it is associated. Quite clearly, the pathogenesis of such injuries is linked to the unique and vulnerable phase of musculoskeletal development of childhood. The presence of areas of bone-cartilage maturation and the relative weakness of growing bones compared with ligaments and tendons are significant factors.

\section{Musculoskeletal growth}

Conventional medical wisdom should remind us that children are simply not little adults. This fact is often ignored by enthusiastic sports administrators, coaches and parents who coax and cajole their pre-adolescent protégés to swifter, higher, stronger competitive levels (Citius, altius, fortius).

The skeletal uniqueness of children is the essence of their physical vulnerability to repetitious microtrauma. Sites of vigorous musculoskeletal development in long bones and their musculotendinous attachments are the specific areas of potential sports injury (Figure 2).

The growth process of bone is well understood. Children are developmentally unique in that they possess a physis or growth plate in the ends of growing long bones. This proliferating plate of cartilage separates the epiphysis (the portion of bone between the physis and the joint surface) from the metaphysis (or remainder of the long bone). The so called epiphyseal plate closure signals fusion of primary and secondary centres of ossification and is recognized clinically as bony maturation. However, if injury occurs before epiphyseal plate closure then 


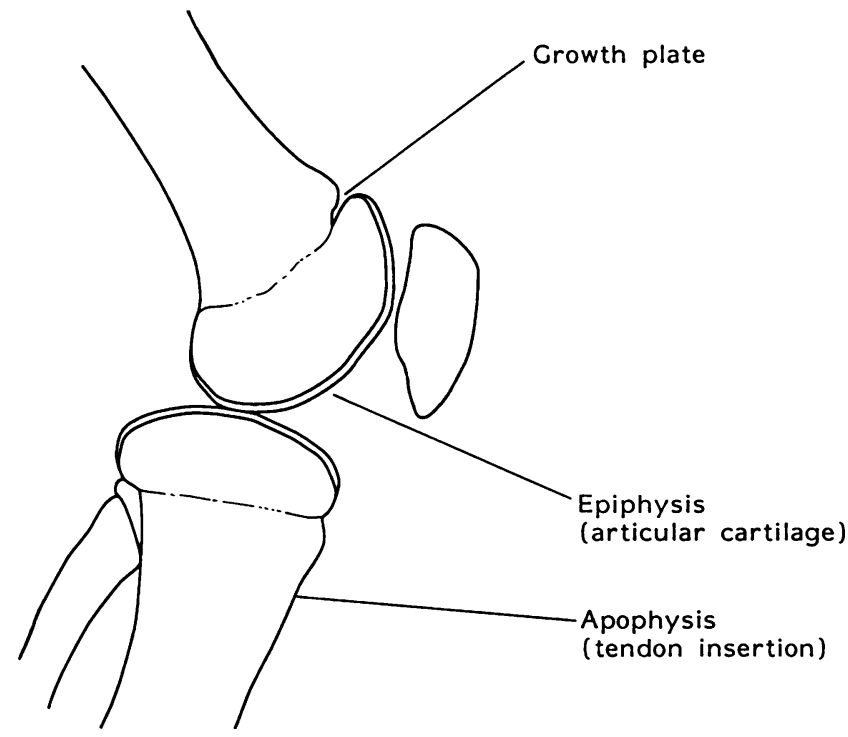

Figure 2. Musculoskeletal growth - sites of potential overuse injury

significant, permanent growth disturbance is likely. Further areas of active growth cartilage exist where muscles and tendons attach to bone. These sites are termed apophyses, vulnerable to repetitive traction forces and often implicated in pre-adolescent overuse injury.
Another significant factor in the genesis of childhood injury is the growth process itself ${ }^{10}$. Musculotendinous units which span growing bones and joints become progressively taut. As a direct consequence, there is a loss of flexibility and an increased potential for traction apophysitis from vigorous or repetitive stress.

In summary, it is evident that the presence of growth cartilage at either the epiphyseal plate, the articular surface of the apophysis predisposes these sites to injury. Asynchronous development of bone and soft tissue provides significant additional risk to the pre-adolescent who, during periods of rapid growth ('spurt') loses flexibility. During such developmental phases it is clinically sensible to reduce the training load imposed upon young athletes.

\section{Sites of injury}

Numerous sites of potential overuse injury are described. Many bear the eponym of the clinician(s) credited with the original description. Others are named for the sport or activity with which they are commonly associated (Table 1). Children's overuse injuries include bursitis, tendonitis, apophysitis of tendon insertions, stress fractures of long bones and, in certain cases, osteochondral injuries of the joint surface $^{10}$. The 'Little League elbow', breaststroker's knee, swimmer's shoulder and jumper's knee reflect

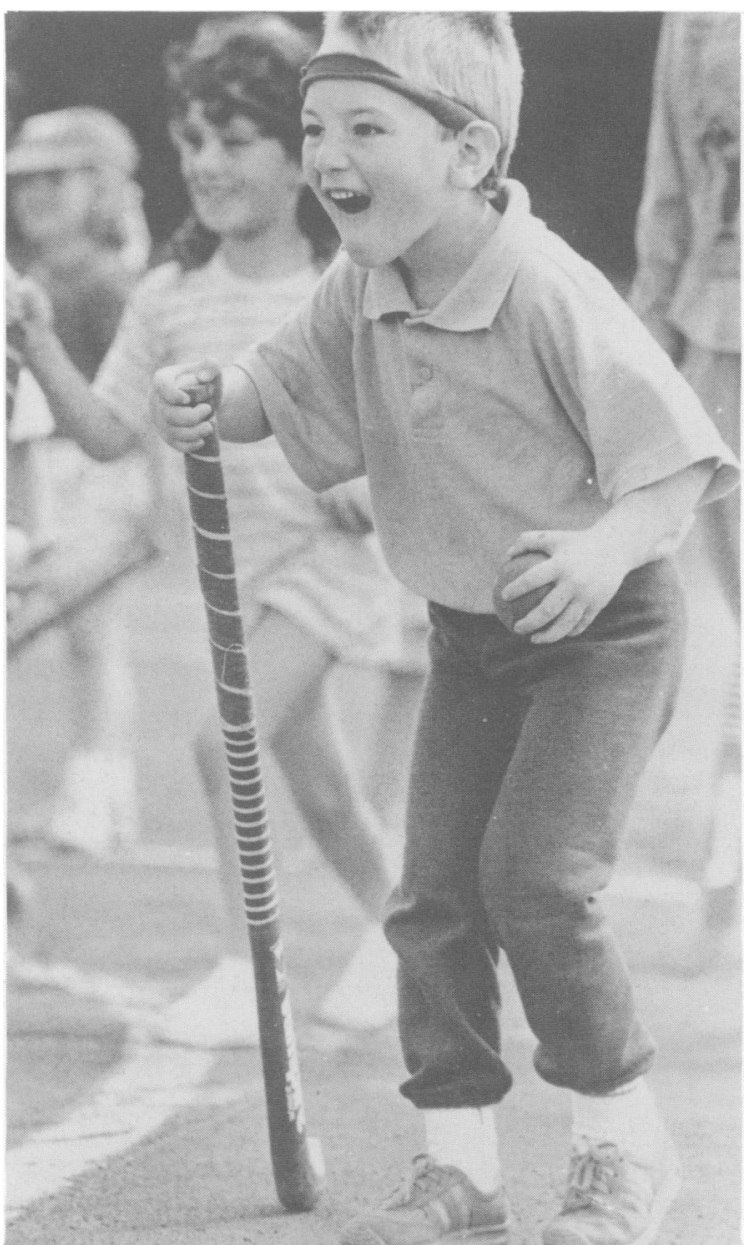


Table 1. Common sites of overuse injury, their associated sports and their popular (eponymous) names ${ }^{8,9}$

\begin{tabular}{lll}
\hline Site & \multicolumn{1}{c}{ Associated sports } & \multicolumn{1}{c}{ Eponym } \\
\hline Tibial tubercle & Jumping, sprinting, skiing, weight training, soccer, horse riding & Osgood-Schlatter's disease \\
Patella (inferior pole) & Soccer, rugby, jumping, skiing, sprinting, dancing & Sinding-Larsen-Johansson syndrome \\
Calcaneus & Running, sprinting, aerobics, soccer, rugby, fencing, dancing & Sever's disease \\
Navicular & Sprinting, running, aerobics, dancing & Köhler's disease \\
Olecranon & Javelin/throwing sports, tennis, gymnastics, weightlifting & Palmer's disease \\
Lunate & Tennis, throwing sports, martial arts & Keinböck's disease \\
Thoracic spine & Rugby, weightlifting, gymnastics, swimming (butterfly) & Scheuermann's disease \\
Second metatarsal head & Dancing, martial arts ('kicking' sports) & Freiberg's disease \\
Third metatarsal head & As for second metatarsal injury & Köhler's II \\
\hline
\end{tabular}

distinct athletic associations. They may be devastating injuries to the pre-adolescent athlete (or his parents), but when recognized early and diagnosed accurately they respond well to conservative treatment and rest.

\section{Back pain}

In a number of activities, but particularly those which demand vigorous repetitive hyperextension of the lumbar spine, low back pain may present. Butterfly swimming, gymnastics and cricket fast bowling are three common examples. Rapid growth of the vertebral bodies is not matched by an increase in the dorsal, tethering soft tissue structures. As a consequence many young athletes have an increased lumbar lordosis. Given the additional stress of repeated hyperextension, children often present with chronic low back ache.

Most investigators agree that the most common causes of such pain are hyperlordotic mechanical low back pain, stress fracture of the pars interarticularis (spondylolysis) or a disc protrusion ${ }^{11}$. Slightly more cephalic pain (thoracolumbar) suggests Scheuermann's disease which appears to be linked to repetitive sporting action. Among the more successful methods of treatment in cases of athletic low back pain are the physiotherapeutic measures of antilordotic exercises, hamstring flexibility and pelvic flexion exercises.

\section{Hip and pelvic pain}

A stable hip and pelvis presides over many athletic activities. In young gymnasts or dancers, hip pain with or without dysfunction is frequently described. Having excluded (radiologically) such problems as slipping of the upper femoral epiphysis or Perthes' osteochondritis of the femoral head, apophysitis at the site of major muscle attachments is a common cause of hip or pelvic pain. There is often focal tenderness in the region of the iliac crest or iliac spines which is accentuated by resisted movement.

\section{Knee pain}

Acknowledged as a common site for impact (macrotrauma) the knee joint is also vulnerable to overuse injury in children. Anterior knee pain may be localized to the patella, its lower pole or the patellar tendon insertion into the tibia. If pain is focal and associated with swelling over the tibial tubercle, the clinical diagnosis is that of Osgood-Schlatter disease. Radiological confirmation is mandatory, as is rest from violent excursions of the knee extensor mechanism.

Retropatellar knee pain, aggravated by running and descending stairs, is strongly suggestive of maltracking of the patella and consequent irritation of the patellofemoral compartment. If the focus of pain is at the lower pole of the patella, a traction apophysitis (Sinding-Larsen-Johansson disease) is likely. Radiographs sometimes demonstrate small bony avulsions which are thought to represent extra ossification centres. Such overuse trauma is best managed with 'active rest' (offering alternative physical options which do not stress the injured part) and frequent clinical review by doctor and/or physiotherapist.

Non-specific knee pain in the young athlete must always be treated with respect. Of the significant conditions to exclude, including referred pain from hip disease, osteochondritis dissecans is important. If an osteochondral fragment becomes separated, there is a significant risk of joint dysfunction and obviously the long-term risk of premature joint degeneration. In such cases a radiograph is mandatory. Of clinical significance is the fact that the presence of osteochondritis dissecans in one site is often linked (through a presumed form of inherited epiphyseal dysplasia) with the same condition in other sites ${ }^{12}$.

\section{Foot and ankle pain}

Heel pain, often described in children playing field sports such as hockey and soccer, is usually worse after activity. Tenderness over the os calcis at the insertion of the tendo Achillis is often associated with Sever's apophysitis. An accompanying tight posterior compartment (gastrocnemius-soleus mechanism) is recognized as part of the developmental cause (reflecting the asynchronous bone versus soft tissue growth rate).

Stress fractures of the os calcis, tarsals and the navicular must be borne in mind when considering 
the causes of foot pain in young athletes. The return from routine radiographs is often small and such techniques as bone scanning and magnetic resonance imaging (MRI) are being vaunted as the most productive diagnostic aids ${ }^{13}$.

\section{Shoulder pain}

Overuse injuries in swimming and throwing events reflect a repetitious pattern of impingement between the humeral head and its rotator cuff attachments (especially supraspinatus) under the coracoacromial arch.

The so called swimmers' shoulder has attracted considerable interest given that thousands of glenohumeral articulations in young performers often results in local, inhibiting pain and dysfunction. Swimmers are found to be weak in external shoulder rotation compared to internal rotation, and this muscle imbalance is thought to be of aetiological importance. Together with the previously described 'impingement' and the relative avascularity of the supraspinatus tendon near its insertion, the pathogenesis of swimmers' shoulder is becoming understood. Treatment suggestions range from rest to biomechanical assessment, physiotherapy modalities, non-steroidal anti-inflammatories and, in some recalcitrant cases, surgical decompression of the structures beneath the coracoacromial arch.

Typical overuse injuries in adults involve bone (stress fractures) tendons and fascia. However, the uniqueness of the young athlete's musculoskeletal system predisposes it to special injury risks. Not only is the young skeleton growing rapidly, but if offers support to developing soft tissues. It follows that the most rapidly developing areas of the child's skeleton are the most vulnerable.

Growth cartilage is present at three important sites: at the growth plate itself (physis); at the joint surface in the form of articular cartilage; and at the site of major muscle-tendon insertions (the apophyses). Insult to these areas of tissue interface results in the typical pre-adolescent injuries of sport. Indiscriminate, repetitive training regimens are associated with pathology at tendinous junctions, tendoperiosteal attachments and immature epiphyseal plates.

It is the responsibility of the coach, administrator and parent to acknowledge the particular susceptibility of young athletes to injury. Medical practitioners, physiotherapists and physical educators have a clinical responsibility to advise, guide and monitor. In accepting that there are numerous factors which contribute to injury a multidisciplinary approach to children in sport will address both the external precipitants (footwear, surfaces, weight training) and the intrinsic factors (growth, muscle-tendon imbalance, flexibility) unique to the immature athlete.

Finally, we must remember that for children's competitive sport to have positive developmental associations, safety and pleasure must be integral elements. The question of skill and the development of social strategies for interaction are important reasons for children to play sport. Those with obvious talent will emerge and if such qualities are nurtured, they will become the stars of the future. The spirit of the 'catch them young' philosophy is tainted by the psychological and physical casualties which result from youngsters being pushed too hard too soon.

\section{References}

1 Thomson RW. Children's sport a sociological perspective. In junior sport - Coaching Association of New Zealand Conference Proceedings. Wellington: NZ Council for Recreation and Sport, 1984: 23-31.

2 Hodge KP. Children in sport: psychological issues. Patient Management. September 1991: 73-5.

3 Rowley S. The Effect of Intensive Training in Young Athletes: A Review of the Research Literature. London: Sports Council, 1986.

4 International Federation of Sports Medicine. Excessive physical training in children and adolescents. N Z J Sports Med 19: 35-6.

5 Stanitski CL. Common injuries in preadolescent and adolescent athletes. Sports Med 1989; 7: 32-41.

6 Clain MR, Hershman EB. Overuse injuries in children and adolescents. Physician and Sportsmedicine 1989; 17: 111-23.

7 Kozar B, Lord RM. Overuse injury in the young athlete: reasons for concern. Physician and Sportsmedicine 1983; 11 116-22.

8 Watson AS. Children in Sport. In: Bloomfield J, Fricker PA, Fitch KD, eds. The Textbook of Science and Medicine in Sport. Melbourne, Australia: Blackwell, 1992: 450, Table 22.3.

9 Swan HAP. Musculoskeletal problems of children in sport. Patient Management June 1991: 71-74, Table 1.

10 Micheli LJ. Paediatric and Adolescent Sports Injuries. Recent Trends in Exercise and Sports Science Reviews. New York, USA: Williams and Wilkins, 1986: 359-74.

11 Micheli LJ. Overuse injuries in children's sports, the growth factor. Orthop Clin North Am 1983; 167: 207-14.

12 Watson AS. Children in sport. In: Bloomfield J, Fricker PA and Fitch KD, eds. The Textbook of Science and Medicine in Sport. Melbourne, Australia: Blackwell, 1992; 436-66.

13 Havránek P. et al. MRI imaging in evaluation of partial growth arrest after physeal injuries in children. J Bone Joint Surg [Am] 1991; 73-A: 1234-41. 